\title{
Experimental infection of conventional newly-weaned piglets with porcine astrovirus
}

\author{
J. C. ULLOA ${ }^{1}$, A. M. OLARTE-APONTE 1 , J. C. OSPINA², M. A. RINCON ${ }^{2}$
}

\begin{abstract}
${ }^{1}$ Laboratorio de Virología, Departamento de Microbiología, Facultad de Ciencias, Grupo de Enfermedades Infecciosas, Pontificia Universidad Javeriana, Bogotá, Colombia; ${ }^{2}$ Laboratorio Nacional de Diagnóstico Veterinario, Instituto Colombiano Agropecuario, Bogotá, Colombia
\end{abstract}

\begin{abstract}
Summary. - The presence of porcine astroviruses in diarrheic and healthy pigs has been reported, however, the consequences of the astrovirus infection during the weaning process have not been described. In this study, eight healthy conventional newly-weaned piglets were used to evaluate effects of astrovirus infection during the first five days. Four piglets were infected with the porcine astrovirus PoAstV/PUJP5 strain and the other four represented the control group. Body weight, rectal temperature, diarrhea and other clinical signs were monitored every 24 hours. The results showed that all animals gained body weight, the occurrence of mild diarrhea on the $3^{\text {rd }}$ day post-infection, and the astroviral presence in diarrheic samples. On the $5^{\text {th }}$ day post-infection all the piglets were euthanized and then intestinal and extra-intestinal tissues were analyzed for the presence of PoAstV/PUJP5. The cytoplasmic antigen of PoAstV/PUJP5 was observed in the enterocytes of infected piglets from jejunum, ileum, colon and in inflammatory cells from mesenteric lymph nodes. In addition, villi atrophy, fusion, epithelial hyperplasia and incipient virus detection in mesenteric lymph were observed. RNAemia could not be proved. This study shows for the first time the effects of porcine astrovirus infection on conventional newly-weaning piglets.
\end{abstract}

Keywords: porcine astrovirus; newly-weaned piglets

\section{Introduction}

Astroviruses are small RNA viruses belonging to the Astroviridae family and have been isolated from several species of mammals (Mamastrovirus) and birds (Avastrovirus). The main pathological presentation associated with these infectious agents is gastrointestinal disease (Appelton and Higgins, 1975; Madeley and Cosgrove, 1975; Shimizu et al., 1990; Bon et al., 1999; Méndez and Arias, 2006); however, the occurrence of hepatitis and fatal nephritis in birds (Ap-

E-mail: julloa@javeriana.edu.co; phone: +57-13208320 Ext: 4029. Abbreviations: dpi = days post-infection; $\mathrm{FFU} / \mathrm{ml}=$ focus forming units per millilitre; hpi = hours post-infection; IP $=$ infected piglets; NIP = non-infected piglets; $\mathrm{ORF}=$ open reading frame; PoAstV $=$ porcine astrovirus; PoAstV/PUJP5 = porcine astrovirus PUJP5 strain; RNA = ribonucleic acid; RT-PCR = reverse transcription PCR pelton and Higgins, 1975; Blomström and Jacobson, 2014) and neurological disorders in some mammals, including pigs, has also been reported (Blomström et al., 2010; Quan et al., 2010; Li et al., 2013; Bouzalas et al., 2014) .

Pathogenesis produced by astrovirus has not been fully characterized. To date, the in vitro approaches have suggested that the mechanism of astrovirus-induced diarrhea is mediated by apoptosis of the infected cells and induction of intestinal epithelial barrier permeability (Schultz-Cherry, 2013). On the other hand, little evidence about the histological changes produced by the astrovirus infection is available; however, it is known that the small intestine is the main target of astroviral replication in both mammals and birds. The few reports of experiments carried out with mammals have shown early effects after astrovirus infection. In gnotobiotic lambs, astrovirus was located $14 \mathrm{~h}$ post-infection (hpi) in highly vacuolated enterocytes in the apical region of the villi; 38 hpi atrophy was identified on the mild villi 
and sloughing of degenerate enterocytes was observed; and 120 hpi the intestines showed a full recovery with mature enterocytes showing no differences with the control. In the case of gnotobiotic calves, the viral infection was located in the dome of enterocytes overlying Peyer's patches at 1-3 days post-infection (dpi). The atrophy of the villi was slight and the viral shedding was confirmed only on the $5^{\text {th }} d p i$ (Meliopoulos and Schultz-Cherry, 2013).

Finally, experiments performed with turkeys revealed an interesting behaviour of the astroviral infection: at $24 \mathrm{hpi}$, viral replication was confirmed in the apical enterocytes, and on the $5^{\text {th }}$ dpi a mild shortening of the villi was observed along with some enterocyte necrosis on the villi base. The infectious virus was isolated from extraintestinal tissues: bursa, thymus, spleen, kidney, skeletal muscle, pancreas and plasma. Additionally, there was no inflammatory response (Meliopoulos and Schultz-Cherry, 2013).

From 2008 onwards, new highly divergent astrovirus strains have been reported (Finkbeiner et al., 2008a,b; 2009a,b) and since then, they have been associated with neurological disorders in humans, minks, cows and recently in pigs (Blomström et al., 2010, 2014; Quan et al., 2010; Li et al., 2013; Bouzalas et al., 2014).

Porcine astrovirus (PoAstV) was reported for the first time in 1980 (Bridger, 1980). As with other hosts, PoAstV has been mainly associated with the production of acute diarrhea in young pigs; however, astrovirus has been detected in the feces and blood serum of diarrheic and healthy conventional pigs of different ages disregarding its effects on newly-weaned piglets. To date, five genotypes of PoAstVs have been reported (Shimizu et al., 1990; Ulloa and Gutierrez, 2010; Brnic et al., 2013; Cai et al., 2016).

It is well known that pigs are susceptible to a variety of viral infections, which in some cases can be transmitted to humans; actually, they are considered a natural reservoir of viruses. The fecal virome of healthy and diarrheic pigs is composed of several families of viruses with a high presence of RNA viruses such as Picornaviridae, Astroviridae, Coronaviridae, Parvoviridae, Circoviridae, Picobirnaviridae, and Caliciviridae (Shan et al., 2011; Amimo et al., 2016). Newly-weaned piglets have been reported to have more coinfections than unweaned piglets (Shan et al., 2011). Whether weaned piglets are susceptible or not to suffering multiple astroviral infections and consequently multiple episodes of diarrhea must be studied. The epidemiological impact of the astroviruses has been low (Schultz-Cherry, 2013), several aspects must be clarified about astroviral infections in pigs such as the effects on the gastrointestinal and nervous systems.

In 2010, the PoAstV/PUJP5 strain was isolated and cell culture-adapted in our laboratory (Ulloa et al., 2011). In order to understand the possible impact of the astrovirus infection on conventional newly-weaned pigs, we carried out an experimental infection using the PoAstV/PUJP5 strain, which has been characterized as highly cytopathic in vitro.

\section{Materials and Methods}

Virus and cells. Embryonic Swine Kidney cells (ESK-4 ATCC ${ }^{\oplus}$ CL-184) were cultured using Advanced DMEM supplemented with $5 \%$ fetal bovine serum (FBS), $2 \mathrm{mmol} / \mathrm{l} \mathrm{L}$-glutamine and antimycotic/antibiotic in a $5 \% \mathrm{CO}_{2}$ atmosphere at $37^{\circ} \mathrm{C}$. When $>90 \%$ confluence was obtained, cells were infected with PoAstV/ PUJP5 previously activated with $100 \mu \mathrm{g} / \mathrm{ml}$ of porcine pancreatic trypsin (Sigma-Aldrich ${ }^{*}$ Cat\# T-4799) for $1 \mathrm{~h}$ at $37^{\circ} \mathrm{C}$ and neutralized with soybean inhibitor (Life Technologies $\%$ Cat\# R-007-100) for $5 \mathrm{~min}$ at room temperature (RT). After 24 hours in a $5 \% \mathrm{CO}_{2}$ atmosphere at $37^{\circ} \mathrm{C}$, the infectious viral particles were harvested using two cycles of freezing $\left(-80^{\circ} \mathrm{C}\right)$ and thawing, followed by centrifugation at $16,100 \mathrm{x} g$ to discard the cellular debris. The supernatant was used to purify the viral particles using $\mathrm{CsCl}$ based on the protocol described previously (Ulloa, 2011) and the particles were suspended in PBS and filtered through a $0.22 \mu \mathrm{m}$ membrane. Finally, the focus forming units per millilitre (FFU/ml) were quantified by immunocytochemistry using ESK-4 cells, a primary polyclonal antibody anti- PoAstV/PUJP5 1:3,000 and goat anti-rabbit IgG 1:3,000 (Invitrogen ${ }^{\text {Tx }} /$ Cat\# 65-6120) / AEC (3-amino-9-ethylcarbazole - Sigma-Aldrich $\%$ Cat\# A5754) as conjugate / substrate with hydrogen peroxide $0.02 \%$. The FFU $/ \mathrm{ml}$ were quantified with an Olympus CKX41 inverted microscope.

Experimental infection of newly-weaned piglets with PoAstV/ PUJP5 and histopathological analysis. Based on the 3Rs (Replacement, Reduction and Refinement), we chose a convenience sample of four animals per group (infected and mock). Thus, eight conventional newly-weaned piglets ( 25 days old - Topigs $®$ ) were purchased from a farm with good management practices. The main criterion for choice of the piglets was the absence of clinical signs associated with diarrhea after a scheme of medication and vaccination against major diseases affecting swines as regulated by the Colombian Livestock and Agricultural Institute (ICA). The animals were transported to an experimental farm, where they were randomly separated into the two groups of four in separate rooms. The feeding protocol was based on the administration of animal feed and sterile water ad libitum. Animals remained for five days in the rooms under environmental adaptation. Body weight and rectal temperature were measured every day. None of the animals showed signs of diarrhea. On the fifth day, blood and fecal samples were obtained from all animals and then, the group 1 was inoculated with sterile PBS and animals of group 2 were inoculated with $2 \times 10^{6} \mathrm{FFU}$ of PoAstV/PUJP5 strain. Piglets were monitored every $24 \mathrm{~h}$ during five days for body weight, rectal temperature and the occurrence of diarrhea and other clinical signs. In addition, blood samples were obtained daily from the jugular vein and the sera separated by centrifugation at $16,000 \mathrm{xg}$ for $15 \mathrm{~min}$ at RT. On the 
fifth day post infection, all piglets were euthanized, and samples of duodenum, jejunum, ileum, colon, liver, brain, kidney, tonsil, lung, heart and mesenteric lymph nodes were obtained and fixed in a $10 \%$ solution of buffered formalin. Each tissue sample was paraffin-embedded to analyze possible microscopic changes associated with the presence of PoAstV/PUJP5. For the analysis of small intestine, a histopathologic scoring scale was applied based on the recommendations reported by Gibson-Corley et al., in order to obtain consistent data (Gibson-Corley et al., 2013). Thus, intestinal tissues from all the non-infected piglets and from three randomly chosen infected piglets were sampled and evaluated.

This study was approved by the Ethical Committee of the Faculty of Sciences, Pontificia Universidad Javeriana - Bogotá, Colombia and carried out according to the established international guidelines (Guidelines for Ethical Conduct in the Care and Use of Nonhuman Animals in Research / http://www.apa.org/science/leadership/care/ guidelines.aspx ).

Diarrhea induced by PoAstV/PUJP5. Feces samples were collected every $24 \mathrm{~h}$ from pens of the infected and non-infected piglets. In order to classify the feces consistency, we used a five-point scale previously reported: 0 , normal, solid and black; 1 , soft brown; 2 , liquid brown; 3, soft yellow; and 4, liquid yellow (Shaw et al., 1995).

Immunohistochemistry. Immunohistochemistry staining was performed according to a conventional protocol (Antibodies: polyclonal anti- PoAstV/PUJP5 1:200 / conjugate goat anti-rabbit IgG / Dako ${ }^{\text {Tx }} /$ Cat\# P0448 1:100) and Meyer's haematoxylin was added as contrast at the end of the procedure.

RT-PCR for PoAstV/PUJP5 and bioinformatics analysis. In order to confirm the presence of the PoAstV/PUJP5 strain after the CsCl purification and in feces and serum, an RT-PCR protocol was used to amplify $417 \mathrm{bp}$ from the ORF2 of the astroviral capsid using the primers AST248F and AST665R, previously standardized in our laboratory (Indik et al., 2006; Ulloa and Gutierrez, 2010). The products amplified were sequenced ambisense by Macrogen, Inc (Seoul, Korea). The sequences obtained were analyzed using the Finch TV program (Geospiza, Inc.; http://www.geospiza.com/ftvdlinfo.html) and, consensus sequences were generated based on binary alignments between sense and antisense sequences with the package CLC Free Sequence Viewer 7 (http://www.clcbio.com/products/ clc-sequence-viewer/). Finally, all sequences were compared with the BLAST program (http://www.ncbi.nlm.nih.gov/blast/Blast.cgi) and included in the Mega 6 program (http://www.megasoftware. net/) in order to make binary alignments with the PoAstV/PUJP5 sequence GenBank Acc. No. FJ160483 obtained in 2010.

\section{Results}

PoAstV/PUJP5 induced mild diarrhea without other apparent signs

Eight domestic newly-weaned piglets were used to evaluate possible effects of an astrovirus infection during

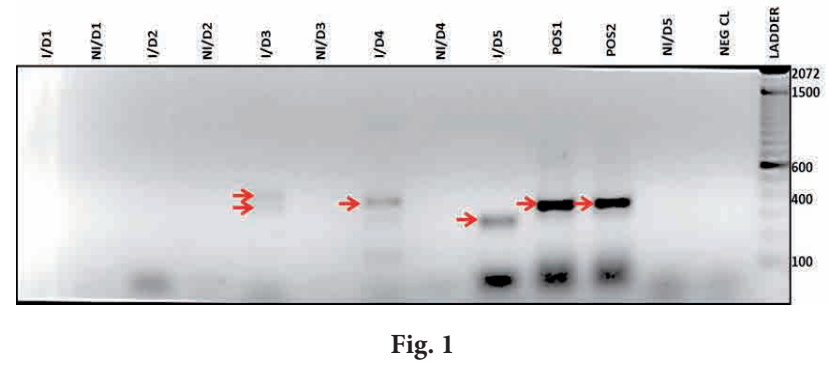

RT-PCR amplification of 417 bp from the ORF2 of PoAstV/PUJP5 from feces samples collected daily in the pens of weaned piglets The red arrows show the fragments amplified from the positive control (PoAstV/PUJP5 CsCl purified - POS1-2) and feces samples collected from infected piglets on the third, fourth and fifth day. Non-specific fragments were amplified from samples collected on the third and fifth day postinfection and one specific fragment on the fourth day. No amplification was observed from the samples collected in the pens of the non-infected piglets. $\mathrm{I}$ = infected; $\mathrm{NI}=$ non-infected; $\mathrm{D} \#=$ day.

the first five days. Half of the animals were orally infected with $2 \times 10^{6} \mathrm{FFU} / \mathrm{ml}$ and the other half were mock-infected.

The induction of diarrhea after the PoAstV/PUJP5 infection was evaluated daily during five days. Feces samples were collected every $24 \mathrm{~h}$ from the pens of the infected and noninfected piglets. The score of the consistency of feces in the group of non-infected animals was 0 during the five days of the experiment and the scores in the group of infected animals were 0 during days 1 and 2, and 2 during days 3,4 and 5 .

In order to associate the PoAstV/PUJP5 with the occurrence of mild diarrhea in the group of infected animals, RT-PCR was used to detect 417 bp of the ORF2, which encodes the viral capsid. The results showed amplification from samples collected from the third to the fifth day; however, the RT-PCR products from the third and fifth days were non-specific but the amplicon obtained from the $4^{\text {th }}$ day was specific (Fig. 1). Samples collected from the noninfected piglets showed no amplification. The test from the RNA extraction was carried out twice and produced equal results. Then, all amplified products with the positive control were sent to sequencing. Only the amplicon obtained on the $4^{\text {th }}$ day could be sequenced and it had a percentage of similarity of $95 \%$ with the previously reported sequence in the GenBank (Acc. No. FJ160483).

On the other hand, body weight and rectal temperature were monitored daily during the five days of the study. In summary, none of the animals had weight loss. Weight gain of non-infected piglet 3 (NIP3) was slight (6.4\%) compared to weight gain of the other piglets of the same group $(\mathrm{NIP} 1=31 \%$; NIP2 $=44.8 \%$; and NIP4 = 16.7\%). Regarding PoAstV/PUJP5 infected animals, their weight gain ranged between $17.6 \%$ and $25 \%$.

Finally, the averages of the rectal temperatures in the non-infected piglets (NIP1, NIP2, NIP3 and NIP4) were 

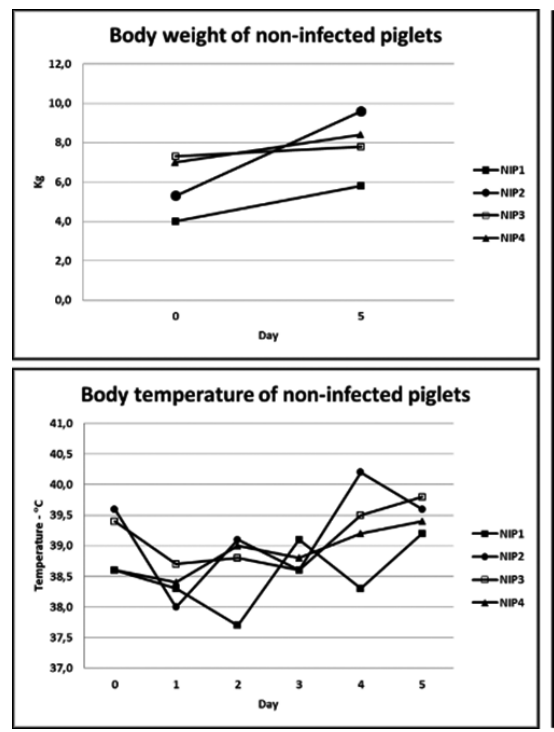

Fig. 2
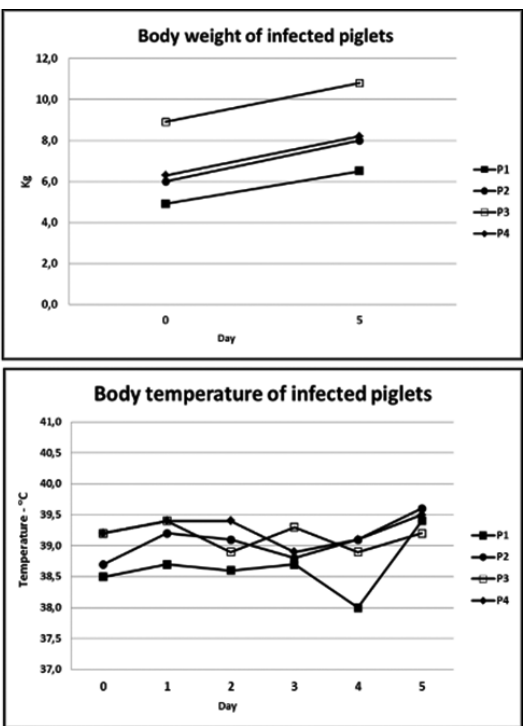

Body weight and rectal temperature during 5 days of weaned domestic piglets infected with PoAstV/PUJP5 None of the animals lost weight. Infected animals had a weight gain between $17.6 \%$ and $25 \%$.

$38.5,39.2,39.1$ and $38.9^{\circ} \mathrm{C}$, respectively; NIP1 had the lowest $\left(37.7^{\circ} \mathrm{C}\right)$ record at day two and NIP2 had the highest $\left(40.2^{\circ} \mathrm{C}\right)$ record at day four. In contrast, infected animals (IP1, IP2, IP3 and IP4) had average temperatures of 38.7, 39.1, 39.2 and $39.3^{\circ} \mathrm{C}$, respectively; the lowest rectal temperature was $38^{\circ} \mathrm{C}$ in IP 1 and the highest $39.6^{\circ} \mathrm{C}$ in the IP2. Figure 2 shows the measures of body weights on the first and fifth days and rectal temperatures during the five days of the study. The normal rectal temperature range in pigs is $38.7-39.8^{\circ} \mathrm{C}$ (Robertshaw, 2004).

PoAstV/PUJP5 detection in intestinal and extra-intestinal tissues and histopathological findings

On the fifth day post-infection the animals were euthanized and several tissue samples were taken and paraffin embedded to detect microscopic lesions associated with the presence of the PoAstV/PUJP5 antigen. Histological findings revealed necrotizing jejunitis with detachment of enterocytes and a strongly positive immunostaining against PoAstV/PUJP5 in the cytoplasm of enterocytes. On the other hand, three of the four animals infected showed a strong signal of the PoAstV/PUJP5 antigen in the cytoplasm of the enterocytes from jejunum, ileum and colon; an incipient detection in cells of mesenteric lymph nodes was observed in two of the infected piglets. Furthermore, none of the tissues evaluated (intestinal or extra-intestinal) from non-infected animals showed the presence of the PoAstV/PUJP5 antigen.

Figure 3 shows the histopathological findings and detection of the PoAstV/PUJP5 capsid antigen in sections of jejunum from infected and non-infected piglets. In addition, Table 1 summarizes the results obtained by conventional immunohistochemistry from the intestinal tissues of all piglets,
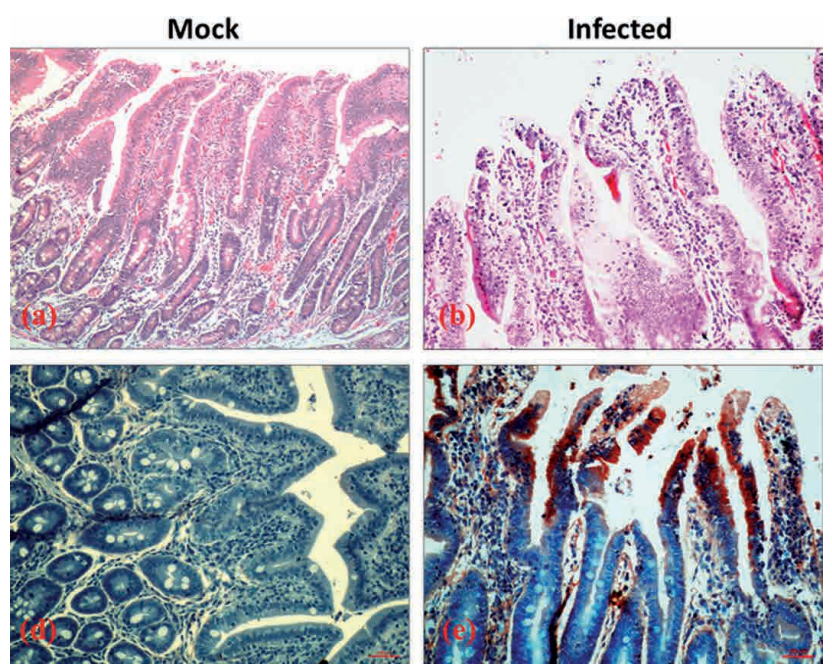

Fig. 3

Histopathology and immunohistochemistry for detection of the PoAstV/PUJP5 capsid antigen in the intestine of experimentally infected piglets

(a) (Jejunum H\&E staining 40x) and (c) (Jejunum immunohistochemistry $200 \mathrm{x})$ represent the mock infection control. Note the absence of lesions in (a) and the negative immunostaining in (c). (b) (Jejunum H\&E 200x) and (d) (Jejunum immunohistochemistry 200x) represent tissues from infected piglets. (b) shows the necrotizing jejunitis with detachment of enterocytes with slight mononuclear inflammation and (d) shows the strongly positive immunostaining against PoAstV/PUJP5 in the cytoplasm of enterocytes. 
Table 1. Results of the immunohistochemistry performed at day 5 post infection to detect PoAstV/PUJP5 capsid antigen in jejunum, ileum, colon and mesenteric lymph nodes from infected domestic weaned piglets

\begin{tabular}{|c|c|c|c|c|}
\hline & Code & Result & Tissue & Site \\
\hline \multirow{4}{*}{$\begin{array}{l}\text { Infected } \\
\text { piglets }\end{array}$} & IP1 & Positive & Colon, jejunum & Cytoplasm of enterocytes \\
\hline & IP2 & Negative & - & - \\
\hline & IP3 & Positive & Ileum, jejunum and mesenteric lymph node & Cytoplasm of enterocytes and cytoplasm of the inflammatory cells \\
\hline & IP4 & Positive & Ileum, colon and mesenteric lymph node & Cytoplasm of enterocytes and incipient in immunological cells \\
\hline \multirow{4}{*}{$\begin{array}{l}\text { Non-infected } \\
\text { piglets }\end{array}$} & NIP1 & Negative & - & - \\
\hline & NIP2 & Negative & - & - \\
\hline & NIP3 & Negative & - & - \\
\hline & NIP4 & Negative & - & - \\
\hline
\end{tabular}

Four animals (IP1-IP4) were orally inoculated with $2 \times 10^{6} \mathrm{FFU}$ of PoAstV/PUJP5 and other four animals (NIP1-NIP4) were inoculated with sterile PBS. The cytoplasmic astroviral antigen detection was observed in all tissues evaluated in three of the infected animals. All non-infected animals were PoAstV/ PUJP5 negative. IP = infected piglets; NIP = non-infected piglets.

Table 2. Histopathological findings in small intestine of weaned piglets experimentally infected and non-infected with PoAstV/PUJP5

\begin{tabular}{|c|c|c|c|c|c|c|c|c|c|c|c|c|c|c|c|}
\hline \multirow[b]{2}{*}{ อิ } & \multicolumn{11}{|c|}{ MUCOSA } & \multicolumn{4}{|c|}{ SUBMUCOSA } \\
\hline & 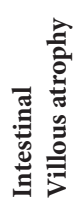 & 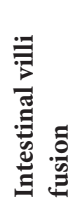 & 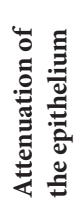 & 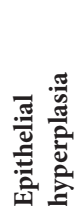 & 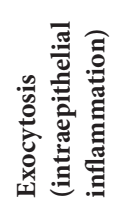 & 量 & 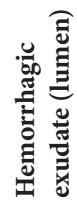 & 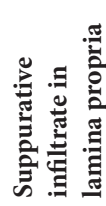 & 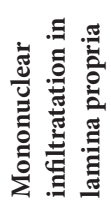 & 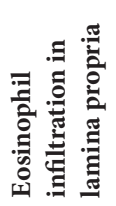 & $\begin{array}{l}\text { Circulatory } \\
\text { changes }\end{array}$ & 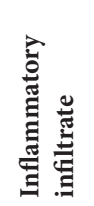 & 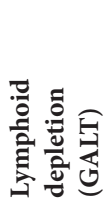 & 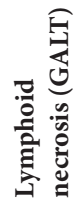 & 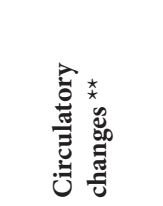 \\
\hline IP1 & $(+)$ & $(-)$ & $(+)$ & $(++)$ & $(++)$ & $(++)$ & $(+)$ & $(-)$ & $(++) \mathrm{L}, \mathrm{P}$ & $(+)$ & Congestion (+) & $(-)$ & $(-)$ & $(-)$ & Edema $(+++)$ \\
\hline IP2 & $(++)$ & $(++)$ & $(-)$ & $(+++)$ & $(++)$ & $(+)$ & $(-)$ & $(-)$ & $(+) \mathrm{L}, \mathrm{P}$ & $(-)$ & Congestion $(+)$ & $(-)$ & $(-)$ & $(-)$ & Edema $(+++)$ \\
\hline IP3 & \multicolumn{15}{|c|}{ NON-INCLUDED } \\
\hline IP4 & $(++)$ & $(++)$ & $(++)$ & $(++)$ & $(+)$ & $(-)$ & $(-)$ & $(-)$ & $(+) \mathrm{L}, \mathrm{P}$ & $(-)$ & Congestion $(+)$ & $(+) \mathrm{L}$ & $(-)$ & $(-)$ & Edema $(++)$ \\
\hline NIP1 & $(+)$ & $(++)$ & $(-)$ & $(++)$ & $(++)$ & $(+)$ & $(-)$ & $(-)$ & $(+) \mathrm{L}, \mathrm{P}$ & $(-)$ & Edema $(+)$ & $(-)$ & $(-)$ & $(-)$ & Edema $(+++)$ \\
\hline NIP2 & $(-)$ & $(-)$ & $(-)$ & $(-)$ & $(-)$ & $(-)$ & $(-)$ & $(-)$ & $(-)$ & $(-)$ & $(-)$ & $(-)$ & $(-)$ & $(-)$ & $(-)$ \\
\hline NIP3 & $(-)$ & $(-)$ & $(-)$ & $(-)$ & $(-)$ & $(-)$ & $(-)$ & $(-)$ & $(-)$ & $(-)$ & $(-)$ & $(-)$ & $(-)$ & $(-)$ & $(-)$ \\
\hline NIP4 & $(+)$ & $(+)$ & $(-)$ & $(-)$ & $(+)$ & $(-)$ & $(-)$ & $(-)$ & $(+) \mathrm{L}, \mathrm{P}$ & $(-)$ & $\begin{array}{l}\text { Congestion and } \\
\text { haemorrhage }(+)\end{array}$ & $(+) \mathrm{L}, \mathrm{P}$ & $(-)$ & $(-)$ & Edema $(++)$ \\
\hline
\end{tabular}

Intestinal tissues from all non-infected piglets and three random samples from three of the infected piglets were evaluated. Severity grade: $(+)$ slight; $(++)$ moderate; $(+++)$ severe; (-) without lesion. L: lymphocytic infiltrate; P: Plasmacytic infiltrate; IP = infected piglets; NIP = non-infected piglets. Histopathologic scoring scale was applied based on the recommendations reported by Gibson-Corley et al. (2013).

where the main finding was the detection of the astroviral antigen in colon and mesenteric lymph nodes.

Other microscopic findings in the small intestine of PoAstV/PUJP5-infected animals included moderate and severe lesions such as: villi atrophy, villi fusion, epithelial hyperplasia, attenuation of the enterocytes, hyperemia, and severe interstitial edema. Furthermore, we observed moderate, mixed and necrotizing colitis with lymphoplasmacytic predominance in the large intestine; mild diffuse alveolar damage in lung; and lymph node depletion in $\mathrm{B}$ and $\mathrm{T}$ zones in the spleen of the infected piglet 1 (IP1); and moderate interstitial mixed pneumonia with lymphoplasmacytic dominance and moderate lymphoid depletion in tonsils of the infected piglet 2 (IP2).
In samples from the small intestine of the non-infected piglet 1 (NIP1) we observed some moderate lesions such as: villi fusion, epithelial hyperplasia, exocytosis and mononuclear infiltration in lamina propria, epithelium attenuation and hyperplasia. Other animals had minor lesions or no bowel injury and two animals had moderate and severe edema. Results are summarized in Table 2.

The RNAemia could not be determined due to lack of clear results.

\section{Discussion}

Astroviruses have been classically characterized by producing a limited gastrointestinal disease in young mammals 
and birds (Schultz-Cherry, 2013). The induction of extraintestinal pathologies in birds (poultry chickens and ducks) such as nephritis and hepatitis and neurological disorders in humans (Quan et al., 2010; Cordey et al., 2016), minks (Blomström et al., 2010), cattle (Li et al., 2013; Bouzalas et al., 2014) and pigs (Blomström et al., 2014) have also been reported. Although it is well known that the Astroviridae family is part of the fecal virome of healthy pigs (Shan et al., 2011; Amimo et al., 2016), it has not been studied whether PoAstV reinfections can produce diarrhea and other signs and lesions in newly-weaned piglets.

Weaning is one of the most stressful events in a pig's life because it can contribute to development of intestinal and immunological disorders that can affect of the pig's health, particularly during the first week after weaning (Campbell et al., 2013). Since the pathogenesis of astrovirus has not been completely studied and the presence of neutralizing antibodies in breast milk has not been reported, the possible reduction of maternal antibodies provided in the milk also could increase the susceptibility to astroviral infections after weaning. If, as a consequence of viral infection, there are delays in growth and other deleterious signs, the pork industry may be affected by economic losses.

The few reports about the pathogenesis of astroviruses have demonstrated that the main target organ in humans, lambs, calves and turkeys is the small intestine, and the most susceptible cells are the villous mature enterocytes of the upper region (Meliopoulos and Schultz-Cherry, 2013). The histological findings obtained in our work correlate partially with previous observations (Meliopoulos and Schultz-Cherry, 2013), where the most susceptible cells of PoAstV/PUJP5 infection were the apical enterocytes of the jejunum accompanied by a cell detachment at $120 \mathrm{hpi}$. However, a necrotizing jejunitis and the infection of ileum, colon and mesenteric lymph nodes were observed in our study. It has been reported that rotavirus can migrate through Peyer's patch, mesenteric lymph node, and peripheral tissues (Mossel and Ramig, 2003). Furthermore, like rotavirus, astroviruses have been reported to produce viremia (Holtz et al., 2011; Brnic et al., 2013). Although in this work we were not able to detect the PoAstV/PUJP5 RNA in sera, the incipient presence of the antigen in the mesenteric lymph node suggests that this organ may be part of the route used by the astrovirus to reach the blood and the central nervous system. It has been reported that those astroviruses that have produced neurological disorders belong to phylogenetically divergent strains (Blomström et al., 2010; Quan et al., 2010; Li et al., 2013; Bouzalas et al., 2014) and still more studies with classical astrovirus are necessary.

The mechanism by which astrovirus produce diarrhea, has not been clarified. The induction of diarrhea after astrovirus infection and its characteristics have been reported as dissimilar in different hosts. In 2-day old gnotobiotic lambs astrovirus produced diarrhea $44 \mathrm{hpi}$ with feces showing a soft consistency and yellow color; in turkeys the induction of a profuse and watery diarrhea at $24 \mathrm{hpi}$ was reported using turkey astrovirus 2 (TAstV-2) and diarrhea continued until 12 dpi with several changes in the intestines. In other animals like dogs and humans astrovirus did not produce diarrhea but in calves astrovirus induced slight changes in feces color and consistency (Meliopoulos and Schultz-Cherry, 2013).

Although in this study we observed the detachment of enterocytes in three of four infected piglets (75\%), a mild diarrhea but not an acute diarrhea was observed. Tissue sampling during necropsy was limited to collecting a single sample of each tissue from each animal at $120 \mathrm{hpi}$, so it may be speculated that the loss of mature enterocytes was occurring only in some regions of the intestine, otherwise acute diarrhea would have been observed. Although in our study we did not evaluate the presence of neutralizing antibodies against PoAstV/PUJP5, the experimental infection was confirmed and observed the loss of enterocytes correlates with the cytopathic effect observed in vitro. Further studies taking a larger number of tissue samples are necessary to clarify this point.

In summary our study confirms the enterotropism (small and large intestines) of porcine astrovirus and shows for the first time some effects produced on conventional newlyweaned piglets after experimental astrovirus infection. Our results also correlate with the low impact reported for astroviruses causing diarrhea in pigs due to the absence of clinical signs such as depression, nausea, vomiting, fever and weight loss. The migration route of astrovirus to extra-intestinal tissues must be studied in order to understand other possible associated diseases such as the nervous system disorders reported by other authors.

Acknowledgments. We thank Pontificia Universidad Javeriana for providing financial support to the project entitled: Evaluación de un modelo in vivo para estudiar la enfermedad producida por astrovirus a través de la infección experimental de lechones con la cepa de astrovirus porcino PUJP5 - No. 00004528. We thank Luis Veloza (DVM) and Mauricio Collazos (DVM) for their assistance in the handling of the animals and the collection of samples.

\section{References}

Amimo JO, El Zowalaty ME, Githae D, Wamalwa M, Djikeng A, Nasrallah GK (2016): Metagenomic analysis demonstrates the diversity of the fecal virome in asymptomatic pigs in East Africa. Arch. Virol. 161, 887-897. https://doi. org/10.1007/s00705-016-2819-6

Appelton H, Higgins, PG (1975): Viruses and gastroenteritis in infants. Lancet 1, 1297. https://doi.org/10.1016/S01406736(75)92581-7

Blomström A, Ley C, Jacobson M (2014): Astrovirus as a possible cause of congenital tremor type AII in piglets? Acta Vet. Scand. 56, 82. https://doi.org/10.1186/s13028-014-0082-y 
Blomström AL, Widén F, Hammer AS, Belák S, Berg M (2010): Detection of a novel astrovirus in brain tissue of mink suffering from shaking mink syndrome by use of viral metagenomics. J. Clin. Microbiol. 48, 4392-4439. https:// doi.org/10.1128/JCM.01040-10

Bon F, Fascia P, Dauvergne M, Tenenbau, D, Planson H, Petion AM, Pothier P, Kohli E (1999): Prevalence of group A rotavirus, human calicivirus, astrovirus, and adenovirus type 40 and 41 infections among children with acute gastroenteritis in Dijon, France. J. Clin. Microbiol. 37, 3055-3058.

Bouzalas I, Wüthrich D, Walland J, Drögemüller C, Zurbriggen A, Vandevelde M, Oevermann A, Bruggmann R, Seuberlich $\mathrm{T}$ (2014): Neurotropic astrovirus in cattle with nonsuppurative encephalitis in europe. J. Clin. Microbiol. 52, 3318-3324. https://doi.org/10.1128/JCM.01195-14

Bridger JC (1980): Detection by electron microscopy of caliciviruses, astroviruses and rotavirus-like particles in the faeces of piglets with diarrhoea. Vet. Rec. 107, 532-533.

Brnic D, Prpic J, Keros T, Roic B, Staresina V, Jemersic L (2013): Porcine astrovirus viremia and high genetic variability in pigs on large holdings in Croatia. Infec. Genet. Evol. 14, 258-264. https://doi.org/10.1016/j.meegid.2012.12.027

Cai Y, Yin W, Zhou Y, Li B, Ai L, Pan M, Guo W (2016): Molecular detection of Porcine astrovirus in Sichuan Province, China. Virol. J. 6, 13-16. https://doi.org/10.1186/s12985015-0462-6

Campbell JM, Crenshaw JD, Polo J (2013): The biological stress of early weaned piglets. J. Anim. Sci. Biotechnol. 4, 19. https://doi.org/10.1186/2049-1891-4-19

Cordey S, Vu DL, Schibler M, L'Huillier AG, Brito F, Docquier M, Posfay-Barbe KM, Petty TJ, Turin L, Zdobnov EM, Kaiser L (2016): Astrovirus MLB2, a New Gastroenteric Virus Associated with Meningitis and Disseminated Infection. Emerg. Infect. Dis. 22, 846-853. https://doi.org/10.3201/ eid2205.151807

Finkbeiner SR, Holtz LR, Jiang Y, Rajendran P, Franz CJ, Zhao G, Kang G, Wang D (2009a): Human stool contains a previously unrecognized diversity of novel astroviruses. Virol. J. 6, 161. https://doi.org/10.1186/1743-422X-6-161

Finkbeiner SR, Kirkwood CD, Wang D (2008a): Complete genome sequence of a highly divergent astrovirus isolated from a child with acute diarrhea. Virol. J. 5, 117-123. https:// doi.org/10.1186/1743-422X-5-117

Finkbeiner SR, Li Y, Ruone S, Conrardy C, Gregoricus N, Toney D, Virgin HW, Anderson LJ, Vinjé J, Wang D, Tong S (2009b): Identification of a Novel Astrovirus (Astrovirus VA1) Associated with an Outbreak of Acute Gastroenteritis. J. Virol. 83, 10836-10839. https://doi.org/10.1128/ JVI.00998-09

Finkbeiner SR, Allred AF, Tarr PI, Klein EJ, Kirkwood CD, Wang D (2008b): Metagenomic analysis of human diarrhea: viral detection and discovery. PLoS Pathog. 4. pp. e1000011. https://doi.org/10.1371/journal.ppat.1000011

Gibson-Corley K, Olivier AK, Meyerholz DK (2013): Principles for valid histopathologic scoring in research. Vet. Pathol. 50, 1007-1015. https://doi.org/10.1177/0300985813485099
Holtz L, Wylie KM, Sodergren E, Jiang Y, Franz CJ, Weinstock GM, Storch GA, Wang D (2011): Astrovirus MLB2 viremia in febrile child. Emerg. Infect. Dis. 17, 2015-2052. https:// doi.org/10.3201/eid1711.110496

Indik S, Valicek 1, Smid B, Dvorakova H, Rodak L (2006): Isolation and partial characterization of a novel porcine astrovirus. Vet. Microbiol. 117, 276-283. https://doi.org/10.1016/j. vetmic.2006.06.020

Li L, Diab S, McGraw S, Barr B, Traslavina R, Higgins R, Talbot T, Blanchard P, Rimoldi G, Fahsbender F, Page B, Phan TG, Wang C, Deng X, Pesavento, P, Delwart E (2013): Divergent Astrovirus Associated with Neurologic Disease in Cattle. Emerg. Infect. Dis. 19, 1385-1392. https://doi. org/10.3201/eid1909.130682

Madeley C, Cosgrove BP (1975): $28 \mathrm{~nm}$ particles in faeces in infantile gastroenteritis. Lancet 2, 451-452. https://doi. org/10.1016/S0140-6736(75)90858-2

Meliopoulos V, Schultz-Cherry S (2013): Astrovirus Pathogenesis. In Schultz-Cherry S (Ed.): Astrovirus Research. Springer Science+Business Media New York, pp. 65-78.

Méndez E, Arias C (2006): Astroviruses. In Knipe D, Howley P, Griffin D, Lamb R, Martin M (Ed.): Fields Virology. Lippincott Williams \& Wilkins, Philadelphia, pp. 982-1000.

Mossel EC, Ramig RF (2003): A lymphatic mechanism of rotavirus extraintestinal spread in the neonatal mouse. J. Virol. 77, 12352-12356. https://doi.org/10.1128/JVI.77.22.12352$\underline{12356.2003}$

Quan P, Wagner TA, Briese T, Torgerson TR, Hornig M, Tashmukhamedova A, Firth C, Palacios G, Baisre-De-Leon A, Paddock CD, Hutchison SK, Egholm M, Zaki SR, Goldman JE, Ochs HD, Lipkin WI (2010): Astrovirus encephalitis in boy with X-linked agammaglobulinemia. Emerg. Infect. Dis. 16, 918-925. https://doi.org/10.3201/ eid1606.091536

Robertshaw D (2004): Temperature Regulation and Thermal Environment. In: Reece WO (Ed.) Dukes' Physiology of Domestic Animals. 12th ed. Cornell University Press.

Schultz-Cherry S (2013): Astrovirus Research: essential ideas, everyday impacts, future directions. Springer New York. https://doi.org/10.1007/978-1-4614-4735-1

Shan T, Li L, Simmonds P, Wang C, Moeser A, Delwart E (2011): The fecal virome of pigs on a high-density farm. J. Virol. 85, 11697-11708. https://doi.org/10.1128/JVI.05217-11

Shaw R, Hempson SJ, Mackow ER (1995): Rotavirus diarrhea is caused by nonreplicating viral particles. J. Virol. 69, 5946-5950.

Shimizu M, Shirai J, Narita M, Yamane, T (1990): Cytopathic astrovirus isolated from porcine acute gastroenteritis in an established cell line derived from porcine embryonic kidney. J. Clin. Microbiol. 28, 201-206.

Ulloa JC, Gutierrez MF (2010): Genomic analysis of two ORF2 segments of new porcine astrovirus isolates and their close relationship with human astroviruses. Can. J. Microbiol. 56, 569-577. https://doi.org/10.1139/W10-042

Ulloa JC, Guzman F, Guerrero CA, Gutierrez MF (2011): Identification of two immunoreactive peptides useful for the detection of Porcine astrovirus. Intervirology 55, 311-317. 